\title{
Aberration-corrected Electron Microscopy Studies of Novel Nanostructures in Highly Aligned Nano-twinned Metals
}

\author{
K. Yoshida, ${ }^{*}$ C. Shute, ${ }^{* *}$ E.D. Boyes, ${ }^{*}$ P.L. Gai, ${ }^{*}$ and J.R. Weertman**
}

* The Nanocentre, Department of Chemistry, Department of Physics and Department of Electronics, The University of York, York Y010 5DD, U.K. (Email: pgb500@york.ac.uk)

** Department of Materials Science and Engineering, Northwestern University, Evanston IL 60208

Nano-twinned metals exhibit remarkable strength and high structural stability [1,2]. Nano-twinned $\mathrm{Cu}$ samples were deformed in tension-tension and compression-compression fatigue, and were then compressed and indented [1]. The high stress tension-tension fatigue and indentation has revealed interesting microstructural changes, mainly de-nanotwinning. To examine what a strong shear stress would do, a small disk of the material was subjected to high pressure torsion (HPT). FIB transverse sections were examined near both surfaces at about $1 \mathrm{~mm}$ from the center in a sample given a half turn. FIB images have shown three distinct regions: a very fine grain size at the surface which ends in a distinct boundary; a section of grains elongated in the shear direction, and near the bottom the twinned regions which look rather irregular (FIG.1a). In order to obtain better insights into the nanostructure of nano-twinned metals at the atomic level for the first time, we have examined the FIB section of nano-twinned metals using a double aberration-corrected (AC) JEOL 2200 FS (S) TEM (AC-TEM) operating at better than 1 Angstrom resolution [3], in the Nanocentre at the University of York, U.K. In addition to the atomic scale imaging of nano-grains and grain boundaries, we have performed high precision chemical composition analyses from the same areas using EDX in the AC-TEM.

Cleaned FIB sample was carefully mounted in the AC-TEM sample holder. AC-TEM data illustrate that near the bottom of the FIB sample more band-like regions are present as shown in FIG.1(b,c and f). FIG.1(f) shows dislocations in one of the bands, terminating at the adjacent boundaries. Preliminary diffraction data carried out on a few bands indicate that they are in a twin relationship. Near the upper region (arrowed) more 'irregular' grain structures (with some elongated grains) were observed as shown in FIG.1(d) and (e). Atomic imaging has shown that intergranular regions consist of interfacial defects and Moire fringes (due to grain overlap). Significantly, these atomic resolution AC-TEM data have demonstrated that the grains in all the regions of the FIB sample are crystalline. Some dislocations are observed within the grains. Our results also reveal that some grains exhibit triple-point intergranular regions. FIG.2 illustrates an atomic resolution image of a crystalline triple point. The three grains (A,B and C) are in [110] orientations with 111 planes in the grain A parallel to the atomic scale (nano)-twin at the at the triple point boundary. The atomic resolution image clearly shows that the triple point region is crystalline and in [110] orientation. Some grains exhibited atomically sharp grain boundaries with no defects. High precision EDX data of the nanograins and the grain boundary regions have shown that they are copper with no discernible impurities. The results are crucial to understanding the nanostructural stability and the strength of highly aligned nano-twinned metals.

\section{References}

[1] J. R. Weertman, C Shute et al, MRS Proceedings (Volume FF), December 2009;

[2] J. R. Weertman et al., Metal and Mat. Trans. 40 (2009) 2256. 
[3] P.L. Gai et al., Microscopy Research and Technique, 72 (2009) 153.

[4] We thank the University of York, Yorkshire Forward/European Union and JEOL for supporting the York-JEOL Nanocentre at the University of York.
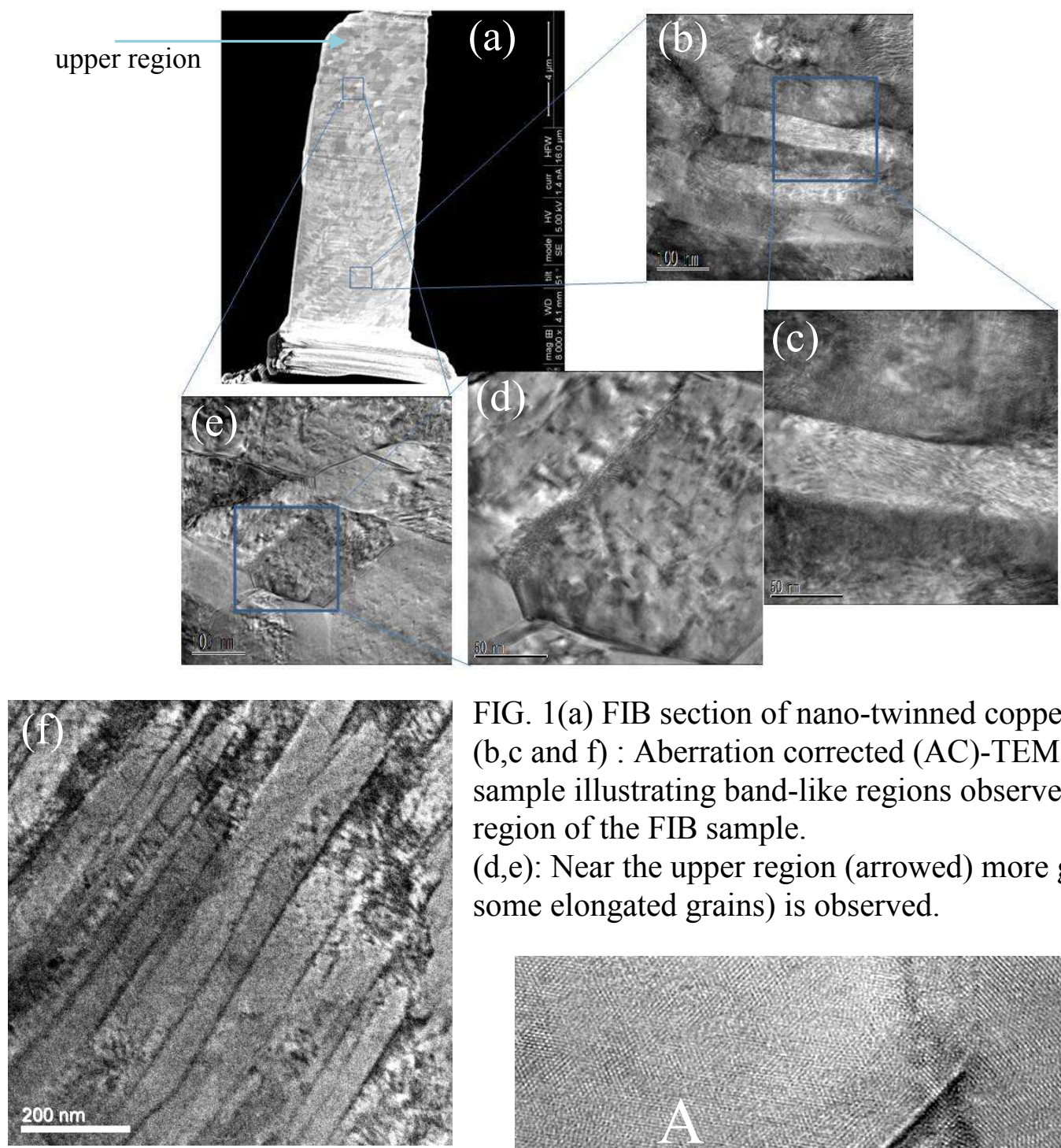

FIG. 1(a) FIB section of nano-twinned copper ;

(b,c and f) : Aberration corrected (AC)-TEM images of the FIB sample illustrating band-like regions observed near the bottom region of the FIB sample.

$(\mathrm{d}, \mathrm{e})$ : Near the upper region (arrowed) more granular structure (with some elongated grains) is observed.

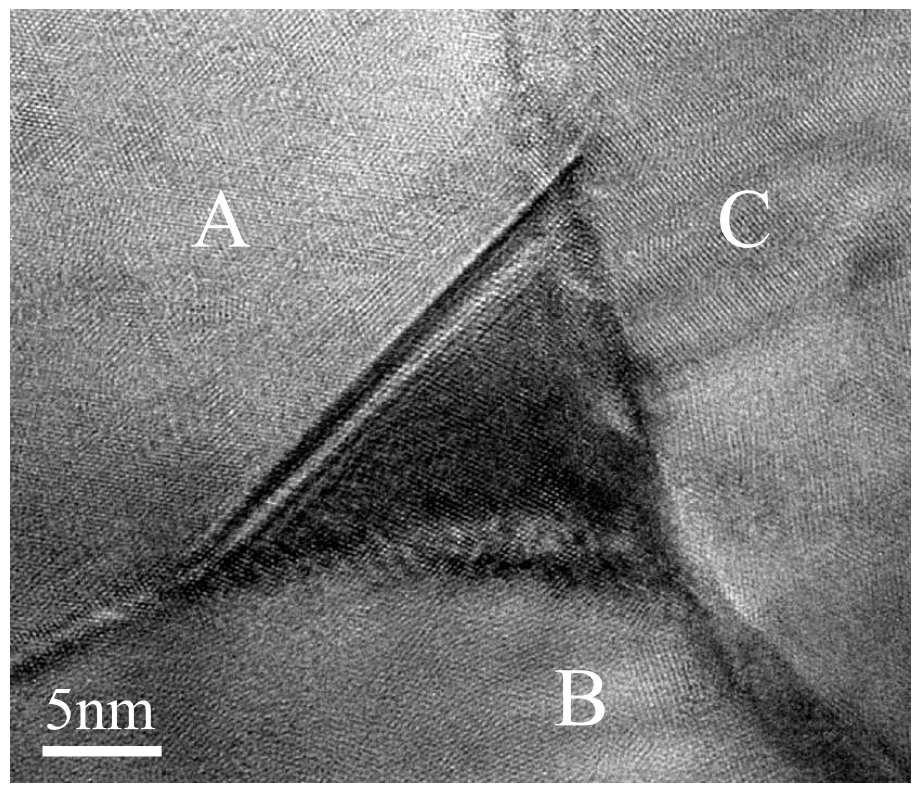

FIG. 2 Aberration-corrected atomic resolution image of a grain boundary triple-point observed from the area in the upper region of the FIB sample. 